\title{
Billion Organisms per Milligram
}

National Cancer Institute

\section{Source}

National Cancer Institute. Billion Organisms per Milligram. NCI Thesaurus. Code C71194.

A unit of measure of organism content expressed in billions of organisms per unit of mass equal to one milligram. 